\title{
Article \\ Impact of Extended and Restricted Antibiotic Deescalation on Mortality
}

\author{
Hwei Lin Teh ${ }^{1, *(\mathbb{D})}$, Sarimah Abdullah ${ }^{2}{ }^{\mathbb{D}}$, Anis Kausar Ghazali ${ }^{2}$, Rahela Ambaras Khan ${ }^{1}$, Anitha Ramadas ${ }^{1}$ and \\ Chee Loon Leong ${ }^{3}$
}

check for updates

Citation: Teh, H.L.; Abdullah, S.; Ghazali, A.K.; Khan, R.A.; Ramadas, A.; Leong, C.L. Impact of Extended and Restricted Antibiotic Deescalation on Mortality. Antibiotics 2022, 11, 22. https://doi.org/ $10.3390 /$ antibiotics 11010022

Academic Editors: Rabia Hussain, Jaya Muneswarao, Zaheer Ud-Din Babar and Rita Murri

Received: 21 October 2021

Accepted: 7 December 2021

Published: 27 December 2021

Publisher's Note: MDPI stays neutral with regard to jurisdictional claims in published maps and institutional affiliations.

Copyright: (C) 2021 by the authors. Licensee MDPI, Basel, Switzerland. This article is an open access article distributed under the terms and conditions of the Creative Commons Attribution (CC BY) license (https:// creativecommons.org/licenses/by/ $4.0 /)$.
1 Pharmacy Department, Hospital Kuala Lumpur, Ministry of Health Malaysia, Kuala Lumpur 50586, Malaysia; rahela.ak@gmail.com (R.A.K.); ramadas.anitha@gmail.com (A.R.)

2 Biostatistics and Research Methodology Unit, Universiti Sains Malaysia (Health Campus), Kota Bharu 16150, Malaysia; sarimah@usm.my (S.A.); anisyo@usm.my (A.K.G.)

3 Infectious Disease Unit, Hospital Kuala Lumpur, Ministry of Health Malaysia, Kuala Lumpur 50586, Malaysia; bkho@hotmail.com

* Correspondence: hwei.lin@moh.gov.my; Tel.: +60-192778091
Abstract: Background: More data are needed about the safety of antibiotic de-escalation in specific clinical situations as a strategy to reduce exposure to broad-spectrum antibiotics. This study aims to compare the survival curve of patient de-escalated (early or late) against those not de-escalated on antibiotics, to determine the association of patient related, clinical related, and pressure sore/device related characteristics on all-cause 30-day mortality and determine the impact of early and late antibiotic de-escalation on 30-day all-cause mortality. Methods: This is a retrospective cohort study on patients in medical ward Hospital Kuala Lumpur, admitted between January 2016 and June 2019. A Kaplan-Meier survival curve and Fleming-Harrington test were used to compare the overall survival rates between early, late, and those not de-escalated on antibiotics while multivariable Cox proportional hazards regression was used to determine prognostic factors associated with mortality and the impact of de-escalation on 30-day all-cause mortality. Results: Overall mortality rates were not significantly different when patients were not de-escalated on extended or restricted antibiotics, compared to those de-escalated early or later $(p=0.760)$. Variables associated with 30-day all-cause mortality were a Sequential Organ Function Assessment (SOFA) score on the day of antimicrobial stewardship (AMS) intervention and Charlson's comorbidity score (CCS). After controlling for confounders, early and late antibiotics were not associated with an increased risk of mortality. Conclusion: The results of this study reinforce that restricted or extended antibiotic deescalation in patients does not significantly affect 30-day all-cause mortality compared to continuation with extended and restricted antibiotics.

Keywords: antibiotic; antimicrobial; de-escalation; streamlining; mortality; outcome; safety

\section{Introduction}

Antibiotic overconsumption and inappropriate antibiotic use remain the key drivers of bacterial resistance, with $30-50 \%$ of prescribed antibiotics being used inappropriately in hospital settings [1,2]. Antimicrobial resistance may result in clinical and economic adverse outcomes and a lack of new and effective antibiotics down the pipeline. Therefore, available broad-spectrum antibiotics must be used judiciously [3]. To address the increasing burden of multi-drug resistant bacterial infections, antimicrobial stewardship (AMS) programs are promoted to rationalize antibiotic prescription and conserve remaining antibiotics while improving patient outcomes. The current effort to improve antibiotic stewardship in Malaysia has been in its early stages since the national protocol on AMS was launched nationwide in 2014 [4]. The antimicrobial stewardship program strongly recommends de-escalation in order to promote judicious antimicrobial use and limit costs, adverse events, and the development of antibiotic resistance [5]. However, it is less commonly 
practiced than desired. Studies have shown that one of the main barriers is uncertainty regarding the safety of de-escalation, despite it being a standard of care among practicing physicians, especially in negative cultures [6,7]. Although the safety of de-escalation has been well established in various international studies, there is currently only one study in Malaysia on antibiotic de-escalation, which focuses on a single infection of ventilatorassociated pneumonia in an intensive care unit [8]. Thus, offering more evidence for the safety of de-escalation will not only increase implementation, but also improve knowledge of the variables influencing the overall outcome of de-escalation. The aim of this study is (i) to compare the survival curves for de-escalation (early and late) and non-de-escalation on extended or restricted antibiotics; (ii) to determine the association of patient-related, clinically related, and pressure sore/device-related characteristics with the all-cause 30-day mortality of patients with suspected bacterial infection initiated on extended or restricted antibiotics; and (iii) to determine the impact of antibiotic de-escalation on all-cause 30-day mortality of patients with suspected bacterial infection initiated on extended and restricted antibiotics. We hypothesized that there would be no difference in survival probabilities between patients not de-escalated on antibiotics and those who had early or late deescalation, while the prognostic factors for all-cause 30-day mortality of patients with suspected bacterial infection initiated with extended or restricted antibiotics would be patient-related, clinically related, and pressure sore/device-related characteristics. We also hypothesized that there would be no significant detrimental impact of early and late de-escalation on all-cause 30-day mortality.

\section{Materials and Methods}

\subsection{Study Design}

This was a retrospective cohort study on patients on extended and restricted antibiotics: Carbapenem (2016-2019) with the addition of patients on vancomycin and colistin (2018-2019) by reviewing medical record files in Hospital Kuala Lumpur. The accrual time for this study was three and half years, from 1 January 2016 to 30 June 2019, with an additional 1 month of follow up from 1 July 2019 to 31 July 2019.

\subsection{Inclusion and Exclusion Criteria}

Patients are included if they are aged $\geq 18$ years old, admitted to the medical ward and started on Carbapenem (meropenem, imipenem/cilastatin, ertapenem), vancomycin, or colistin. Patients should also be reviewed by the antimicrobial stewardship team (AMS team) and deemed suitable for de-escalation. This AMS team, which consists of members recommended by Ministry of Health Malaysia (infectious disease physician, clinical pharmacists, clinical microbiologist, and an infection control nurse), will be prompted on cases initiated with Carbapenem, vancomycin, and colistin in medical wards. All such cases were reviewed Thursday of every week by the AMS team, and recommendation of de-escalation is communicated verbally directly to the primary treating team, who has the final decision on whether to accept the recommendation of de-escalation. Exclusion criteria of this study are those whose survival is less than $24 \mathrm{~h}$ after septic workup were drawn, if treatment was changed to another broader spectrum antibiotic (escalation of antibiotic), or if the patient was transferred in from another institution.

\subsection{Data Collection}

A data collection form was used to record all required information retrieved manually from patient's medical file located in medical records. Such information included patient related characteristics, clinical related characteristics, pressure sore or device related characteristics, and if de-escalation has been performed.

\subsection{Variables and Definition}

The primary outcome of interest in this study was the event-death from all causes. Death status was verified by referring to death certificate in medical records retrieved 
from hospital archive center. The survival time was defined as the duration from the initiation of extended or restricted antibiotic to the date of the event. Patients still alive at study closure were censored on 31 July 2019. Antibiotic de-escalation is defined by changing an initially appropriate antimicrobial therapy from an empirical broad-spectrum characteristic to a narrower-spectrum one (by either changing the antimicrobial agent or by discontinuing an eventual antimicrobial combination, or both) according to culture results or clinical conditions, or shortening of the time course of the antimicrobial therapy, or withholding antibiotics. The classification and ranking of antibiotics was developed by consensus [9]. Early de-escalation was defined as de-escalation occurring within 4 days while late de-escalation was defined as de-escalation occurring beyond 4 days of extended or restricted antibiotic initiation. Censored was defined as alive or loss to follow up at day 30 days post antibiotic (extended or restricted antibiotic) initiation. Comorbidity defined as a pre-existing disease or condition in addition to the disease or condition designated as the principal diagnosis. The pre-existing disease had to be an active problem in one of two ways. Either the disease required treatment during the hospital admission, or the disease had permanently altered some organ function. Antimicrobial therapy administered before the susceptibility results were available was considered empirical. Therapy administered after microbiological report was considered microbiologically directed therapy. Indwelling catheter included temporary/permanent central venous device and percutaneous drainage. Source of infection in each patient was standardized according to Centre for Disease Control (CDC) criteria [10]. Sepsis severity was assessed using the Sequential Organ Failure Assessment (SOFA) [11,12]. Multidrug-resistant isolates were those producing Extended Spectrum $\beta$-lactamases (ESBLs) or AmpC $\beta$-lactamases, or Carbapenem-resistant. Investigations taken on Day 0 are investigations taken on the day of extended/restricted antibiotic initiation, or up to a maximum $48 \mathrm{~h}$ before initiation of extended/restricted antibiotic.

\subsection{Statistical Analysis}

Survival analysis was carried out by Kaplan-Meier survival curves and analysed by the Fleming-Harrington test for the first objective. Besides the Kaplan-Meier survival curve, simple univariable and multivariable Cox regression analysis was performed for the second and third objective. The regression coefficient (b) with standard error (SE), adjusted hazard ratio (AHR) with its 95\% confidence interval, Wald statistics and its corresponding $p$ value were reported. Variables with $p$ value $<0.25$ were selected to be included in multivariable analysis. Methods used for the selection of variables to be included in the model are forced entry, forward stepwise, and backward stepwise. In this process, the probability of entry $(\mathrm{Pe})$ and the probability of removal $(\mathrm{Pr})$ are pre-determined as 0.05 and 0.1 , respectively, throughout the whole variable selection process. The preliminary final model was checked for multicollinearity, specification error, and proportionality of hazard assumption. Data analysis was performed using STATA SE Version 14. The sample size required for this study to have an $80 \%$ power to detect a $70 \%$ difference survival time of de-escalated vs. non-de-escalated group with a two-sided test with an a level of 0.05 was calculated to be 172 . The $70 \%$ difference in survival time was based on expert opinion of an infectious disease consultant as previous studies on the safety of de-escalation were largely undertaken with logistic statistical analysis and no data on difference in median survival time were readily available. Sample size was calculated using power and sample size calculation (PS) Software.

\section{Results}

A total of 180 subjects fulfilled the inclusion criteria, and because the sample size calculated approximates sampling frame no probability sampling was applied in this study. All 180 subjects were included in the final analysis, and all subjects completed follow-up. Overall, there were 62 deaths (34.4\%) and 118 censored events (65.6\%). The 118 subjects were censored because death did not occur at the end of follow-up. Out of 180 patients seen by the AMS team, $132(73.3 \%)$ cases were successfully de-escalated on extended or 
restricted antibiotics, of which 79 patients (43.9\%) had early de-escalation while 53 patients $(29.4 \%)$ had late de-escalation. The main de-escalation was discontinuation of extended and restricted antibiotic $(37.8 \%)$, followed by changing to a narrow spectrum antibiotic $(31.7 \%)$ and shortening of the duration of antibiotic therapy (3.8\%). Patient characteristics, clinical characteristics, pressure sore or device related characteristics between the groups of de-escalation are shown in Tables 1 to 3. Simple and multiple survival regression analyses of patient, clinical, and pressure sore or devise related variables are shown in Tables 4 to 7 .

Table 1. Frequency distribution of patient related characteristics based on de-escalation group.

\begin{tabular}{|c|c|c|c|c|}
\hline $\begin{array}{l}\text { Patient Related } \\
\text { Characteristics }\end{array}$ & $\begin{array}{c}\text { No De-Escalation } \\
n=48 \\
\text { Frequency }(\%)\end{array}$ & $\begin{array}{c}\text { Early De-Escalation } \\
\qquad \begin{array}{l}n=79 \\
\text { Frequency }(\%)\end{array}\end{array}$ & $\begin{array}{c}\text { Late De-Escalation } \\
\qquad \begin{array}{c}n=53 \\
\text { Frequency }(\%)\end{array}\end{array}$ & $p$-Value \\
\hline \multicolumn{5}{|l|}{ Age } \\
\hline Age $\leq 65$ years & $30(26.6)$ & $50(44.2)$ & $33(29.2)$ & \multirow{2}{*}{0.992} \\
\hline Age $>65$ years & $18(26.9)$ & $29(43.3)$ & $20(29.8)$ & \\
\hline \multicolumn{5}{|l|}{ Gender } \\
\hline Male & $20(22.0)$ & $40(44.0)$ & $31(34.0)$ & \multirow{2}{*}{0.240} \\
\hline Female & $28(31.5)$ & $39(43.8)$ & $22(24.7)$ & \\
\hline \multicolumn{5}{|l|}{ Ethnicity } \\
\hline Malay & $22(23.7)$ & $41(44.1)$ & $30(32.2)$ & \multirow{4}{*}{0.464} \\
\hline Chinese & $12(30.0)$ & $14(35.0)$ & $14(35.0)$ & \\
\hline Indian & $9(25.7)$ & $19(54.3)$ & $7(20.0)$ & \\
\hline Others & $5(42.0)$ & $5(42.0)$ & $2(16.0)$ & \\
\hline \multicolumn{5}{|l|}{ ICU Stay } \\
\hline No & $39(27.7)$ & $66(46.8)$ & $36(25.5)$ & \multirow{2}{*}{0.087} \\
\hline Yes & $9(23.1)$ & $13(33.3)$ & $17(43.6)$ & \\
\hline \multirow{2}{*}{\multicolumn{5}{|c|}{$\begin{array}{l}\text { Invasive Mechanical } \\
\text { Ventilation }\end{array}$}} \\
\hline & & & & \\
\hline No & $32(29.1)$ & $51(46.4)$ & $27(24.5)$ & \multirow[b]{2}{*}{0.190} \\
\hline Yes & $16(22.9)$ & $28(40.0)$ & $26(37.1)$ & \\
\hline \multicolumn{5}{|l|}{ CCS } \\
\hline $0-2$ & $30(27.3)$ & $45(40.9)$ & $35(31.8)$ & \multirow[b]{2}{*}{0.562} \\
\hline$\geq 3$ & $18(25.7)$ & $34(48.6)$ & $18(25.7)$ & \\
\hline \multicolumn{5}{|l|}{ McCabe Score } \\
\hline 1 & $41(25.2)$ & $70(42.9)$ & $52(31.9)$ & \multirow[b]{2}{*}{0.068} \\
\hline$\geq 2$ & $7(41.2)$ & $9(52.9)$ & $1(5.9)$ & \\
\hline \multicolumn{5}{|l|}{ Illicit drug use } \\
\hline No & $48(27.3)$ & $76(43.2)$ & $52(29.5)$ & \multirow[b]{2}{*}{0.364} \\
\hline Yes & $0(0.00)$ & $3(75.0)$ & $1(25.0)$ & \\
\hline \multicolumn{5}{|l|}{ Smoking status } \\
\hline Non smoker & $37(28.2)$ & $56(42.8)$ & $38(29.0)$ & \multirow{3}{*}{0.928} \\
\hline Ex-smoker & 4 (19.1) & $10(47.6)$ & $7(33.3)$ & \\
\hline Active smoker & $7(25.0)$ & $13(46.4)$ & $8(28.6)$ & \\
\hline \multicolumn{5}{|c|}{$\begin{array}{l}\text { History of Hospital Admission } \\
\text { within } 3 \text { months }\end{array}$} \\
\hline No & $33(27.1)$ & $53(43.3)$ & $36(29.5)$ & \multirow{2}{*}{0.981} \\
\hline Yes & $15(25.9)$ & $26(44.8)$ & $17(29.3)$ & \\
\hline \multicolumn{5}{|c|}{$\begin{array}{l}\text { History of antibiotic exposure } \\
\text { within } 3 \text { months }\end{array}$} \\
\hline No & $38(29.7)$ & $50(39.0)$ & $40(31.2)$ & \multirow[b]{2}{*}{0.113} \\
\hline Yes & $10(19.2)$ & $29(55.8)$ & $13(25.0)$ & \\
\hline \multicolumn{5}{|l|}{ Presence of ESRF } \\
\hline No & $47(27.3)$ & 75 (43.6) & $50(29.1)$ & \multirow[b]{2}{*}{0.642} \\
\hline Yes & $1(12.5)$ & $4(50.0)$ & $3(37.5)$ & \\
\hline
\end{tabular}


Table 1. Cont.

\begin{tabular}{|c|c|c|c|c|}
\hline $\begin{array}{l}\text { Patient Related } \\
\text { Characteristics }\end{array}$ & $\begin{array}{c}\text { No De-Escalation } \\
n=48 \\
\text { Frequency }(\%)\end{array}$ & $\begin{array}{c}\text { Early De-Escalation } \\
n=79 \\
\text { Frequency }(\%)\end{array}$ & $\begin{array}{c}\text { Late De-Escalation } \\
\begin{array}{c}n=53 \\
\text { Frequency }(\%)\end{array}\end{array}$ & $p$-Value \\
\hline \multicolumn{5}{|l|}{$\begin{array}{l}\text { Diabetes with end } \\
\text { organ failure }\end{array}$} \\
\hline No & $35(26.5)$ & $59(44.7)$ & $38(28.8)$ & \multirow{2}{*}{0.928} \\
\hline Yes & $13(27.1)$ & $20(41.7)$ & $15(31.2)$ & \\
\hline \multicolumn{5}{|l|}{ Presence of HIV } \\
\hline No & $48(27.1)$ & $76(42.9)$ & $53(30.0)$ & \multirow{2}{*}{0.142} \\
\hline Yes & $0(0.00)$ & $3(100.0)$ & $0(0.00)$ & \\
\hline \multicolumn{5}{|c|}{ Presence of Malignancy } \\
\hline No & $45(27.8)$ & $69(42.6)$ & $48(29.6)$ & \multirow{2}{*}{0.499} \\
\hline Yes & $3(16.7)$ & $10(55.6)$ & $5(27.8)$ & \\
\hline
\end{tabular}

Table 2. Frequency distribution of clinical related characteristics based on de-escalation group.

\begin{tabular}{|c|c|c|c|c|}
\hline Clinical Related Characteristics & $\begin{array}{c}\text { No De-Escalation } \\
n=48 \\
\text { Frequency }(\%)\end{array}$ & $\begin{array}{c}\text { Early De-Escalation } \\
\qquad \begin{array}{l}n=79 \\
\text { Frequency }(\%)\end{array}\end{array}$ & $\begin{array}{l}\text { Late De-Escalation } \\
\qquad \begin{array}{l}n=53 \\
\text { Frequency }(\%)\end{array}\end{array}$ & $p$-Value \\
\hline \multicolumn{5}{|l|}{ Acquisition of infection } \\
\hline Community acquired & $26(26.3)$ & $42(42.4)$ & $31(31.3)$ & \multirow{2}{*}{0.826} \\
\hline $\begin{array}{l}\text { Hospital or healthcare } \\
\text { acquired }\end{array}$ & $22(27.2)$ & $37(45.7)$ & $22(27.1)$ & \\
\hline \multicolumn{5}{|l|}{$\begin{array}{l}\text { Extended or Restricted antibiotic } \\
\text { initiated }\end{array}$} \\
\hline Meropenem & $36(29.5)$ & $47(38.5)$ & $39(32.0)$ & \multirow{5}{*}{0.672} \\
\hline Imipenem & $4(16.0)$ & $14(56.0)$ & $7(28.0)$ & \\
\hline Ertapenem & $6(23.1)$ & $14(53.9)$ & $6(23.0)$ & \\
\hline Colistin & $1(25.0)$ & $2(50.0)$ & $1(25.0)$ & \\
\hline Vancomycin & $1(33.3)$ & $2(66.7)$ & $0(0.00)$ & \\
\hline \multicolumn{5}{|l|}{ Therapy of antibiotic } \\
\hline Empirical & $35(30.7)$ & $45(39.5)$ & $34(29.8)$ & \multirow[b]{2}{*}{0.193} \\
\hline $\begin{array}{l}\text { Microbiologically } \\
\text { directed }\end{array}$ & $13(19.7)$ & $34(51.5)$ & $19(28.8)$ & \\
\hline \multicolumn{5}{|l|}{ Source of infection } \\
\hline Others & $32(32.0)$ & $38(38.0)$ & $30(30.0)$ & \multirow[b]{2}{*}{0.122} \\
\hline Respiratory & $16(20.0)$ & $41(51.2)$ & $23(28.8)$ & \\
\hline \multicolumn{5}{|l|}{ Aetiology (sterile culture) } \\
\hline No growth & $41(28.9)$ & $59(41.5)$ & $42(29.6)$ & \multirow{3}{*}{0.658} \\
\hline Others & $4(19.1)$ & $12(57.1)$ & $5(23.8)$ & \\
\hline Klebsiella pneumonia & $3(23.0)$ & $5(38.5)$ & $5(38.5)$ & \\
\hline \multicolumn{5}{|l|}{ Resistance (sterile culture) } \\
\hline Sensitive strain or Others & $3(23.1)$ & $9(69.2)$ & $1(7.7)$ & \multirow{2}{*}{0.082} \\
\hline $\begin{array}{l}\text { Multidrug resistant } \\
\text { isolate }^{\mathrm{a}}\end{array}$ & $4(19.1)$ & $8(38.0)$ & $9(42.9)$ & \\
\hline \multicolumn{5}{|l|}{$\mathrm{CRP}(\mathrm{mg} / \mathrm{L})$ on Day $0^{\mathrm{b}}$} \\
\hline $25-64$ & $10(32.3)$ & $13(41.9)$ & $8(25.8)$ & \multirow{4}{*}{0.662} \\
\hline $65-143$ & $5(20.0)$ & $9(36.0)$ & $11(44.0)$ & \\
\hline $144-240$ & $2(13.3)$ & $7(46.7)$ & $6(40.0)$ & \\
\hline$>240$ & $3(33.3)$ & $5(41.7)$ & $3(25.0)$ & \\
\hline \multicolumn{5}{|l|}{ Temperature $\left({ }^{\circ} \mathrm{C}\right)$ on Day 0} \\
\hline$\leq 37.5$ & $23(31.5)$ & $28(38.4)$ & $22(30.1)$ & \multirow{2}{*}{0.376} \\
\hline$>37.5$ & $25(23.4)$ & $51(47.6)$ & $31(29.0)$ & \\
\hline
\end{tabular}


Table 2. Cont.

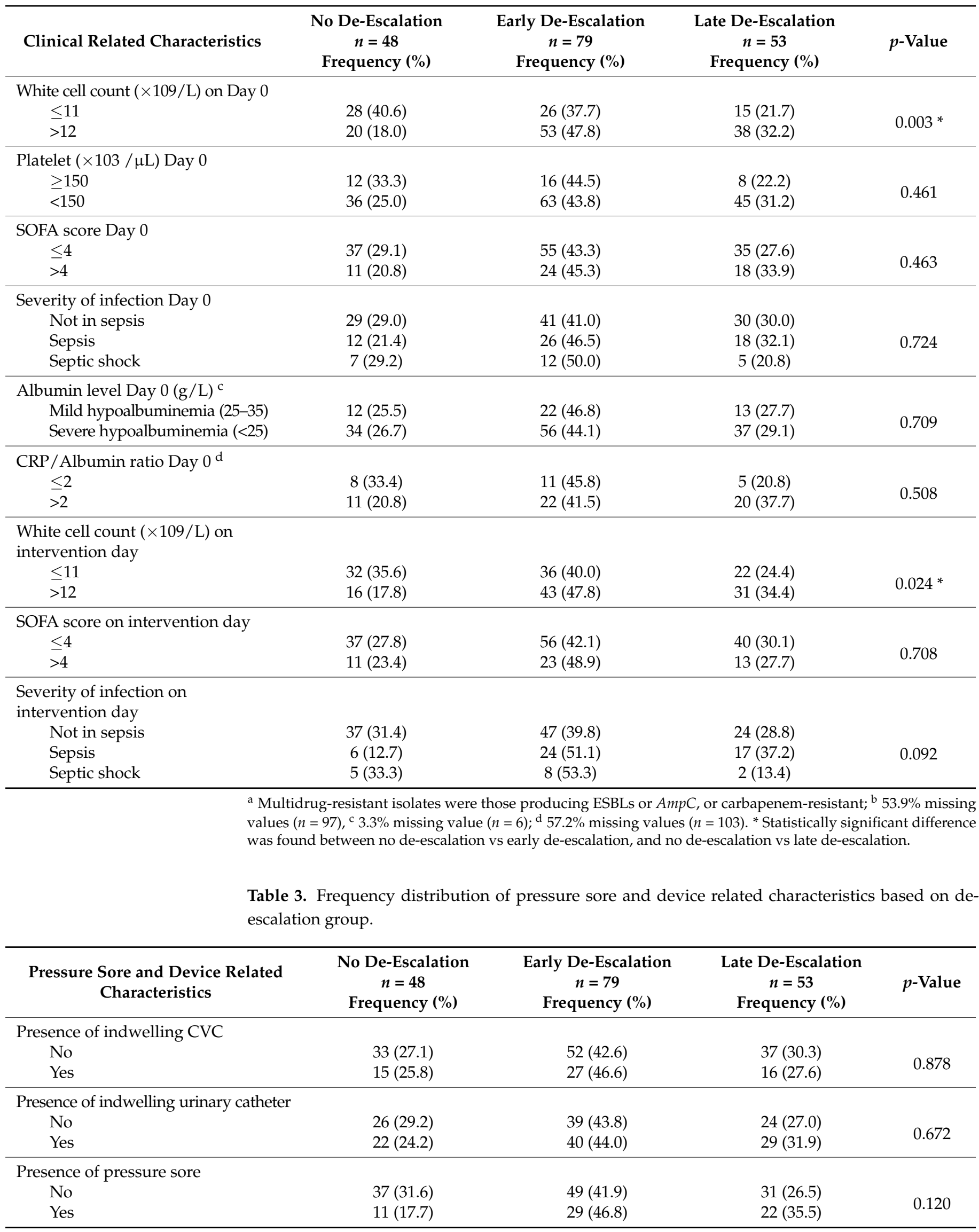


Table 4. Patient related factor of all-cause 30-days mortality in patients suspected with bacterial infection on extended or restricted antibiotic using simple Cox proportional hazards regression model $(n=180)$.

\begin{tabular}{|c|c|c|c|c|c|c|}
\hline Variables & $\begin{array}{c}\text { Event } \\
n=62, \\
\text { Frequency (\%) }\end{array}$ & $\begin{array}{c}\text { Censored } \\
n=118, \\
\text { Frequency }(\%)\end{array}$ & b (SE) & $\begin{array}{c}\text { Crude Hazards } \\
\text { Ratio } \\
(95 \% \text { CI })\end{array}$ & Wald Statistic & $p$-Value \\
\hline \multicolumn{7}{|l|}{ Age } \\
\hline Age $\leq 65$ years & $36(58.1)$ & $77(65.3)$ & 0 & 1 & & \\
\hline Age $>65$ years & $26(41.9)$ & $41(34.7)$ & $0.20(0.26)$ & $1.22(0.74-2.03)$ & 0.77 & 0.441 \\
\hline \multicolumn{7}{|l|}{ Gender } \\
\hline Male & $30(48.4)$ & $61(51.7)$ & 0 & 1 & & \\
\hline Female & $32(51.6)$ & $57(48.3)$ & $0.05(0.25)$ & $1.06(0.64-1.74)$ & 0.21 & 0.832 \\
\hline \multicolumn{7}{|l|}{ Ethnicity } \\
\hline Malay & $27(43.6)$ & $66(55.9)$ & 0 & 1 & & \\
\hline Chinese & $15(24.2)$ & $25(21.2)$ & $0.48(0.32)$ & $1.62(0.85-3.08)$ & 1.47 & 0.142 \\
\hline Indian & $15(24.2)$ & $20(16.7)$ & $0.51(0.33)$ & $1.67(0.88-3.17)$ & 1.57 & 0.117 \\
\hline Others & $5(8.0)$ & $7(6.2)$ & $0.52(0.49)$ & $1.68(0.64-4.40)$ & 1.06 & 0.289 \\
\hline \multicolumn{7}{|l|}{ ICU Stay } \\
\hline No & $11(17.7)$ & $29(24.6)$ & 0 & 1 & & \\
\hline Yes & $51(82.3)$ & $89(75.4)$ & $-0.39(0.35)$ & $0.68(0.34-1.34)$ & 0.26 & 0.264 \\
\hline \multicolumn{7}{|l|}{ Invasive Mechanical } \\
\hline \multicolumn{7}{|l|}{ Ventilation } \\
\hline No & $34(54.8)$ & $36(30.5)$ & 0 & 1 & & \\
\hline Yes & $28(45.2)$ & $82(69.5)$ & $0.87(0.26)$ & $2.38(1.43-3.94)$ & 3.35 & 0.001 \\
\hline \multicolumn{7}{|l|}{ CCS } \\
\hline $0-2$ & $28(45.2)$ & $82(69.5)$ & & 1 & & \\
\hline$\geq 3$ & $34(54.8)$ & $36(30.5)$ & $0.84(0.26)$ & $2.32(1.40-3.86)$ & 3.26 & 0.001 \\
\hline \multicolumn{7}{|l|}{ McCabe Score } \\
\hline 1 & $51(82.3)$ & $112(94.9)$ & 0 & 1 & & \\
\hline$\geq 2$ & $11(17.7)$ & $6(5.1)$ & $0.81(0.34)$ & $2.26(1.17-4.37)$ & 2.41 & 0.016 \\
\hline \multicolumn{7}{|l|}{ Illicit drug use } \\
\hline No & $59(95.2)$ & 117 (99.2) & 0 & 1 & & \\
\hline Yes & $3(4.8)$ & $1(0.8)$ & $1.11(0.59)$ & $3.03(0.95-9.73)$ & 1.87 & 0.062 \\
\hline \multicolumn{7}{|l|}{ Smoking status } \\
\hline Non smoker & $44(71.0)$ & $87(73.7)$ & 0 & 1 & & \\
\hline Ex-smoker & 7 (11.3) & $14(11.9)$ & $0.14(0.41)$ & $1.15(0.51-2.56)$ & 0.34 & 0.737 \\
\hline Active smoker & $11(17.7)$ & $17(14.4)$ & $0.18(0.34)$ & $1.20(0.62-2.33)$ & 0.54 & 0.590 \\
\hline \multirow{3}{*}{\multicolumn{7}{|c|}{$\begin{array}{l}\text { History of Hospital } \\
\text { Admission within } \\
3 \text { months }\end{array}$}} \\
\hline & & & & & & \\
\hline & & & & & & \\
\hline No & $44(71.0)$ & $78(66.1)$ & 0 & 1 & & \\
\hline Yes & $18(29.0)$ & $40(33.9)$ & $-0.17(0.28)$ & $0.84(0.49-1.46)$ & -0.60 & 0.547 \\
\hline \multicolumn{7}{|l|}{$\begin{array}{l}\text { History of antibiotic } \\
\text { exposure within } \\
3 \text { months }\end{array}$} \\
\hline No & $46(74.2)$ & $82(69.5)$ & 0 & 1 & & \\
\hline Yes & $16(25.8)$ & $36(30.5)$ & $-0.16(0.29)$ & $0.85(0.48-1.50)$ & -0.56 & 0.575 \\
\hline \multicolumn{7}{|l|}{ Presence of ESRF } \\
\hline No & 57 (91.9) & 115 (97.5) & 0 & 1 & & \\
\hline Yes & $5(8.1)$ & $3(2.54)$ & $0.87(0.47)$ & $2.38(0.95-5.97)$ & 1.86 & 0.063 \\
\hline \multicolumn{7}{|l|}{$\begin{array}{l}\text { Diabetes with end } \\
\text { organ failure }\end{array}$} \\
\hline No & $39(63.9)$ & $93(78.8)$ & 0 & 1 & & \\
\hline Yes & $23(37.1)$ & $25(21.2)$ & $0.60(0.27)$ & $1.82(1.08-3.06)$ & 2.26 & 0.024 \\
\hline
\end{tabular}


Table 4. Cont.

\begin{tabular}{|c|c|c|c|c|c|c|}
\hline Variables & $\begin{array}{c}\text { Event } \\
n=62, \\
\text { Frequency (\%) }\end{array}$ & $\begin{array}{c}\text { Censored } \\
n=118, \\
\text { Frequency }(\%)\end{array}$ & b (SE) & $\begin{array}{c}\text { Crude Hazards } \\
\text { Ratio } \\
(95 \% \text { CI })\end{array}$ & Wald Statistic & $p$-Value \\
\hline \multicolumn{7}{|l|}{ Presence of HIV } \\
\hline No & $59(95.2)$ & $118(100.0)$ & 0 & 1 & & \\
\hline Yes & $3(4.8)$ & $0(0.0)$ & $1.46(0.60)$ & $4.31(1.34-13.81)$ & 2.46 & 0.014 \\
\hline \multicolumn{7}{|l|}{$\begin{array}{l}\text { Presence of } \\
\text { Malignancy }\end{array}$} \\
\hline No & $52(83.9)$ & $110(93.2)$ & 0 & 1 & & \\
\hline Yes & $10(16.1)$ & $8(6.8)$ & $0.89(0.35)$ & $2.42(1.22-4.80)$ & 2.55 & 0.011 \\
\hline \multicolumn{7}{|c|}{ Chronic liver failure } \\
\hline No & $54(87.1)$ & $114(96.6)$ & 0 & 1 & & \\
\hline Yes & $8(12.9)$ & $4(3.4)$ & 1.03 & $2.80(1.33-5.90)$ & 2.70 & 0.007 \\
\hline
\end{tabular}

Table 5. Clinical related factor of all-cause 30-days mortality in patients suspected with bacterial infection on extended or restricted antibiotic using simple Cox proportional hazards regression model $(n=180)$.

\begin{tabular}{|c|c|c|c|c|c|c|}
\hline Variables & $\begin{array}{c}\text { Event } \\
n=62 \\
\text { Median (IQR)/ } \\
\text { Frequency (\%) }\end{array}$ & $\begin{array}{c}\text { Censored } \\
\quad n=118 \\
\text { Median (IQR)/ } \\
\text { Frequency (\%) }\end{array}$ & b (SE) & $\begin{array}{c}\text { Crude } \\
\text { Hazards Ratio } \\
(95 \% \text { CI })\end{array}$ & $\begin{array}{l}\text { Wald } \\
\text { Statistic }\end{array}$ & $\begin{array}{c}p- \\
\text { Value }\end{array}$ \\
\hline \multicolumn{7}{|l|}{ Acquisition of infection } \\
\hline Community acquired & $25(40.3)$ & $74(62.7)$ & 0 & 1 & & \\
\hline $\begin{array}{l}\text { Hospital or healthcare } \\
\text { acquired }\end{array}$ & $37(59.7)$ & $44(37.3)$ & $0.60(0.26)$ & $1.83(1.09-3.06)$ & 2.30 & 0.022 \\
\hline \multicolumn{7}{|l|}{ Therapy of antibiotic } \\
\hline Empirical & $47(75.8)$ & $67(56.8)$ & 0 & 1 & & \\
\hline $\begin{array}{l}\text { Microbiologically } \\
\text { directed }\end{array}$ & $15(24.2)$ & $51(43.2)$ & $-0.64(0.30)$ & $0.53(0.29-0.95)$ & -2.14 & 0.033 \\
\hline $\begin{array}{l}\text { Duration of Extended or } \\
\text { Restricted antibiotic }\end{array}$ & $4(4) *$ & $5(5) *$ & $-0.05(0.04)$ & $0.95(0.88-1.02)$ & -1.49 & 0.137 \\
\hline \multicolumn{7}{|l|}{ Source of infection } \\
\hline Non respiratory & $27(43.6)$ & $73(61.9)$ & 0 & 1 & & \\
\hline Respiratory & $35(56.4)$ & $45(38.1)$ & $0.56(0.26)$ & $1.76(1.06-2.91)$ & 2.20 & 0.028 \\
\hline \multicolumn{7}{|l|}{ Aetiology (sterile culture) } \\
\hline No growth & $51(82.2)$ & $91(79.8)$ & 0 & 1 & & \\
\hline Others & $7(11.3)$ & $18(12.3)$ & $-0.01(0.40)$ & $0.99(0.45-2.19)$ & -0.02 & 0.981 \\
\hline Klebsiella Pneumonia & $4(6.5)$ & $9(7.9)$ & $-0.29(0.52)$ & $0.97(0.35-2.70)$ & -0.06 & 0.955 \\
\hline \multicolumn{7}{|l|}{ Resistance (sterile culture) } \\
\hline Sensitive strain or Others & $4(36.4)$ & $9(39.1)$ & 0 & 1 & & \\
\hline Multidrug resistant isolate $^{\mathrm{a}}$ & $7(63.6)$ & $14(60.9)$ & $0.10(0.63)$ & $1.11(0.32-3.78)$ & 0.87 & 0.872 \\
\hline \multicolumn{7}{|l|}{$\mathrm{CRP}(\mathrm{mg} / \mathrm{L})$ on Day $0^{\mathrm{b}}$} \\
\hline $25-64$ & $8(40.0)$ & $23(36.5)$ & 0 & 1 & & \\
\hline $65-143$ & $5(25.0)$ & $20(31.7)$ & -0.09 & $0.91(0.29-2.88)$ & -0.15 & 0.878 \\
\hline $144-240$ & $5(25.0)$ & $10(15.9)$ & 0.45 & $1.58(0.50-4.98)$ & 0.78 & 0.434 \\
\hline$>240$ & $2(10.0)$ & $10(15.9)$ & -0.32 & $0.73(0.15-3.51)$ & -0.40 & 0.693 \\
\hline \multicolumn{7}{|l|}{ Temperature $\left({ }^{\circ} \mathrm{C}\right)$ on Day 0} \\
\hline$\leq 37.5$ & $22(35.5)$ & $51(43.2)$ & 0 & 1 & & \\
\hline$>37.5$ & $40(64.5)$ & $67(56.8)$ & $0.30(0.27)$ & 1.35 (0.80-2.27) & 1.13 & 0.257 \\
\hline
\end{tabular}


Table 5. Cont.

\begin{tabular}{|c|c|c|c|c|c|c|}
\hline Variables & $\begin{array}{c}\text { Event } \\
n=62 \\
\text { Median (IQR)/ } \\
\text { Frequency (\%) }\end{array}$ & $\begin{array}{c}\text { Censored } \\
n=118 \\
\text { Median (IQR)/ } \\
\text { Frequency (\%) }\end{array}$ & b (SE) & $\begin{array}{c}\text { Crude } \\
\text { Hazards Ratio } \\
(95 \% \text { CI })\end{array}$ & $\begin{array}{l}\text { Wald } \\
\text { Statistic }\end{array}$ & $\begin{array}{c}p- \\
\text { Value }\end{array}$ \\
\hline \multicolumn{7}{|l|}{$\begin{array}{l}\text { White cell count }(\times 109 / \mathrm{L}) \\
\text { on Day } 0\end{array}$} \\
\hline$\leq 11$ & $28(45.2)$ & $41(34.8)$ & 0 & 1 & & \\
\hline$>12$ & $34(54.8)$ & $77(65.2)$ & $-0.22(0.26)$ & $0.80(0.48-1.32)$ & -0.87 & 0.383 \\
\hline \multicolumn{7}{|l|}{ Platelet $(\times 103 / \mu \mathrm{L})$ Day 0} \\
\hline$\geq 150$ & $22(35.5)$ & $14(11.9)$ & 0 & 1 & & \\
\hline$<150$ & $40(64.5)$ & $104(88.1)$ & $-0.88(0.27)$ & $0.41(0.25-0.70)$ & -3.30 & 0.001 \\
\hline \multicolumn{7}{|l|}{ Albumin level Day $0(\mathrm{~g} / \mathrm{L})^{\mathrm{c}}$} \\
\hline \multicolumn{6}{|l|}{ Mild hypoalbuminemia } & 0.014 \\
\hline $\begin{array}{l}\text { Severe hypoalbuminemia } \\
(<25)\end{array}$ & $52(83.9)$ & $75(67.0)$ & $0.89(0.36)$ & $2.43(1.20-4.93)$ & & \\
\hline \multicolumn{7}{|l|}{ SOFA score Day 0} \\
\hline$\leq 4$ & $26(42.0)$ & $101(85.6)$ & 0 & 1 & & \\
\hline$>4$ & $36(58.0)$ & $17(14.4)$ & $1.63(0.26)$ & $5.11(3.06-8.54)$ & 6.22 & $<0.001$ \\
\hline \multicolumn{7}{|l|}{ Severity of infection Day 0} \\
\hline Not in sepsis & $15(24.2)$ & $85(72.0)$ & 0 & 1 & & \\
\hline Sepsis & $31(50.0)$ & $25(21.2)$ & $1.56(0.33)$ & $4.77(2.57-8,87)$ & 4.95 & $<0.001$ \\
\hline Septic shock & $16(25.8)$ & $8(6.8)$ & $1.80(0.37)$ & $6.01(3.00-12.21)$ & 4.96 & $<0.001$ \\
\hline \multicolumn{7}{|l|}{$\mathrm{CRP} /$ Albumin ratio Day $0^{\mathrm{d}}$} \\
\hline$\leq 2$ & $8(40.0)$ & $16(28.1)$ & 0 & 1 & & \\
\hline$>2$ & $12(60.0)$ & 41 (71.9) & -0.11 & $0.89(0.43-1.84)$ & -0.31 & 0.756 \\
\hline \multicolumn{7}{|l|}{$\begin{array}{l}\text { White cell count }(\times 109 / \mathrm{L}) \\
\text { on intervention day }\end{array}$} \\
\hline$\leq 11$ & $28(45.2)$ & $62(52.5)$ & 0 & 1 & & \\
\hline$>11$ & $34(54.8)$ & $56(47.5)$ & $1.17(0.26)$ & $3.21(1.94-5.31)$ & 4.53 & $<0.001$ \\
\hline \multicolumn{7}{|l|}{$\begin{array}{l}\text { SOFA score on AMS } \\
\text { intervention day }\end{array}$} \\
\hline$\leq 4$ & $25(73.9)$ & $108(91.5)$ & 0 & 1 & & \\
\hline$>4$ & $47(26.1)$ & $10(8.5)$ & $1.96(0.27)$ & $7.10(4.22-11.95)$ & 7.38 & $<0.001$ \\
\hline \multicolumn{7}{|l|}{$\begin{array}{l}\text { Severity of infection on } \\
\text { intervention day }\end{array}$} \\
\hline Not in sepsis & $19(30.7)$ & $99(83.9)$ & 0 & 1 & & \\
\hline Sepsis & $30(48.3)$ & $17(14.4)$ & $1.70(0.30)$ & $5.47(3.06-9.75)$ & 5.75 & $<0.001$ \\
\hline Septic shock & $13(20.0)$ & $2(1.69)$ & $2.13(0.37)$ & $8.44(4.12-17.29)$ & 5.83 & $<0.001$ \\
\hline
\end{tabular}

* IQR: Interquartile range; ${ }^{a}$ Multidrug-resistant isolates were those producing ESBLs or $A m p C$, or carbapenemresistant; ${ }^{\mathrm{b}} 53.9 \%$ missing values $(n=97),{ }^{\mathrm{c}} 3.3 \%$ missing value $(n=6) ;{ }^{\mathrm{d}} 57.2 \%$ missing values $(n=103)$. 
Table 6. Pressure sore and device related factor of all-cause 30-days mortality in patients suspected with bacterial infection on extended or restricted antibiotic using simple Cox proportional hazards regression model $(n=180)$.

\begin{tabular}{|c|c|c|c|c|c|c|}
\hline Variables & $\begin{array}{c}\text { Event } \\
n=62, \\
\text { Frequency (\%) }\end{array}$ & $\begin{array}{c}\text { Censored } \\
n=118 \\
\text { Frequency }(\%)\end{array}$ & b (SE) & $\begin{array}{l}\text { Crude Hazards } \\
\text { Ratio }(95 \% \text { CI })\end{array}$ & Wald Statistic & $\begin{array}{c}p- \\
\text { Value }\end{array}$ \\
\hline \multicolumn{7}{|c|}{ Presence of indwelling CVC } \\
\hline No & $26(42.0)$ & $96(81.4)$ & 0 & 1 & & \\
\hline Yes & $36(58.0)$ & $22(18.6)$ & $1.32(0.26)$ & $3.73(2.24-6.22)$ & 5.04 & $<0.001$ \\
\hline \multicolumn{7}{|c|}{$\begin{array}{l}\text { Presence of indwelling } \\
\text { urinary catheter }\end{array}$} \\
\hline No & $16(25.8)$ & $73(61.9)$ & 0 & 1 & & \\
\hline Yes & $46(74.2)$ & $45(38.1)$ & $1.18(0.29)$ & $3.25(1.84-5.74)$ & 4.05 & $<0.001$ \\
\hline \multicolumn{7}{|c|}{ Presence of pressure sore } \\
\hline No & $29(47.5)$ & $88(74.6)$ & 0 & 1 & & \\
\hline Yes & $32(52.5)$ & $30(25.4)$ & 0.92 & $2.50(1.50-4.15)$ & 3.53 & $<0.001$ \\
\hline
\end{tabular}

Table 7. Antimicrobial stewardship team related intervention on all-cause 30-days mortality in patients suspected with bacterial infection on extended or restricted antibiotic using simple Cox proportional hazards regression $(n=180)$.

\begin{tabular}{|c|c|c|c|c|c|c|}
\hline Variables & $\begin{array}{c}\text { Event } \\
n=62 \\
\text { Frequency } \\
\quad(\%)\end{array}$ & $\begin{array}{c}\text { Censored } \\
n=118 \\
\text { Frequency } \\
\quad(\%)\end{array}$ & b (SE) & $\begin{array}{l}\text { Crude Hazards } \\
\text { Ratio }(95 \% \text { CI) }\end{array}$ & $\begin{array}{c}\text { Wald } \\
\text { Statistic }\end{array}$ & $\begin{array}{c}p \text { - } \\
\text { Value }\end{array}$ \\
\hline \multicolumn{7}{|l|}{ Types of intervention } \\
\hline No de-escalation & $18(29.0)$ & $30(25.4)$ & 0 & 1 & & \\
\hline Early de-escalation & $28(45.2)$ & $51(43.2)$ & $-0.13(0.31)$ & $0.87(0.48-1.60)$ & -0.43 & 0.670 \\
\hline Late de-escalation & $16(25.8)$ & $37(31.4)$ & $-0.31(0.35)$ & $0.73(0.37-1.45)$ & -0.89 & 0.373 \\
\hline \multicolumn{7}{|l|}{ Types of de-escalation } \\
\hline No de-escalation & $18(29.0)$ & $30(25.4)$ & 0 & 1 & & \\
\hline Discontinuation & $23(37.1)$ & $45(38.2)$ & $0.27(1.41)$ & $1.32(0.08-21.03)$ & 0.19 & 0.846 \\
\hline Changing to narrow spectrum & $19(30.7)$ & $38(32.2)$ & $1.31(1.15)$ & $3.70(0.38-35.57)$ & 1.13 & 0.257 \\
\hline Shorten duration & $2(3.2)$ & $5(4.2)$ & $0.86(1.01)$ & $2.37(0.32-17.26)$ & 0.85 & 0.393 \\
\hline
\end{tabular}

\subsection{Survival Curve of Those De-Escalated and Non-De-Escalated on Antibiotics}

In Fleming-Harrington test, the overall mortality rates were not significantly different when patient was not de-escalated on extended or restricted antibiotics, to those de-escalated early or later $(p=0.760)$. Figure 1 show graphical illustration of Kaplan-Meier survival curve between the de-escalation group. 


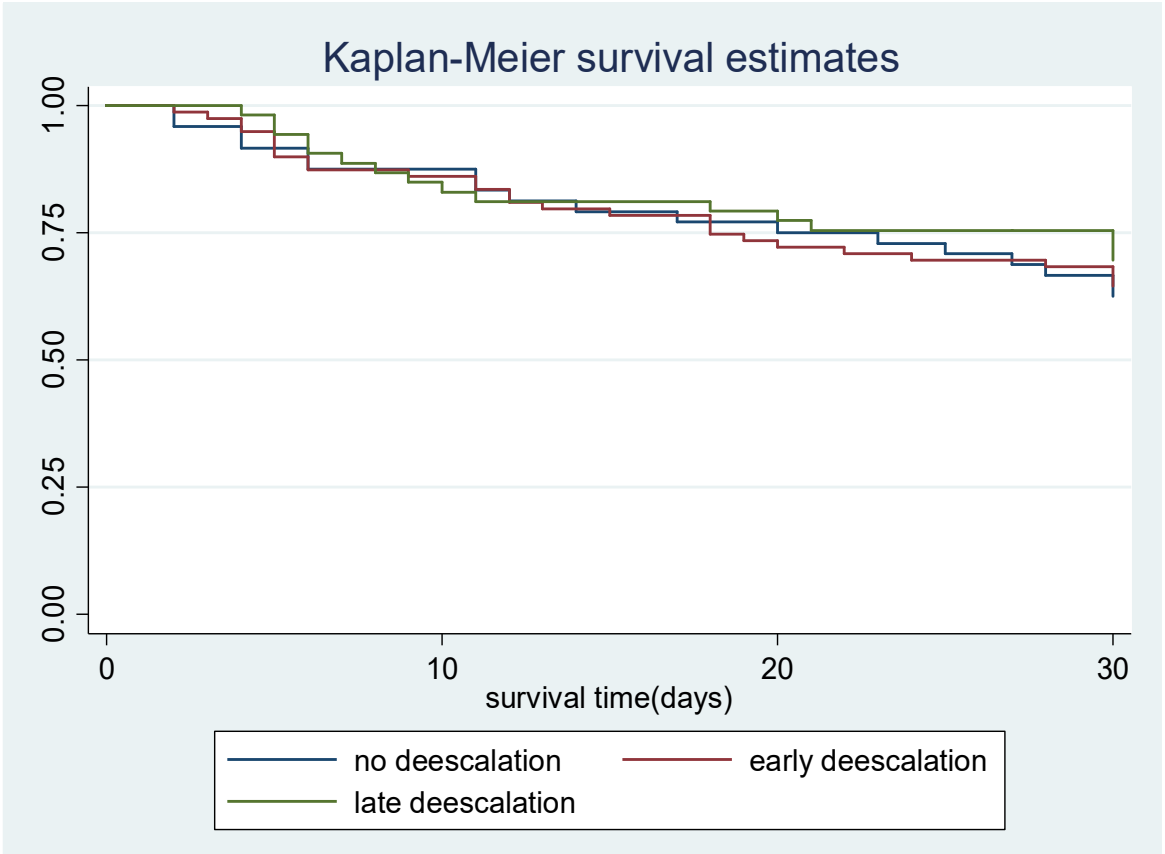

Figure 1. Kaplan-Meier estimates for overall survival rates based on de-escalation group; no deescalation, early de-escalation and late de-escalation.

\subsection{Variables Associated with All Cause 30-Days Mortality}

The univariable analysis of variables associated with all cause 30-days mortality is outlined in Tables 4 to 7 . Multivariable analysis associated with all cause 30-days mortality were Sequential Organ Function Assessment (SOFA) score on the day of antimicrobial stewardship (AMS) intervention (AHR 6.61, 95\% CI 3.90,11.18; $p<0.001$ ) and Charlson's comorbidity score (AHR 1.97, 95\% CI 1.17,3.30; $p=0.01$ ). Multicollinearity and interactions were not observed. The preliminary final model was properly specified (Table 8). Hazard function plots, Log-minus-log plots, Schoenfeld partial residual plots, as well as scaled and non-scaled Schoenfeld residuals test indicated proportionality of hazard. Regression diagnostics were performed by Cox-Snell residual analysis, which indicated that the model is a good fit, while Harrell's C statistic was calculated to assess the discrimination ability of the preliminary final model. The C-statistics was 0.795 , suggesting acceptable discrimination.

Table 8. Univariable and multivariable analysis of prognostic factor for 30-day all-cause mortality of patients with suspected bacterial infection on extended or restricted antibiotics.

\begin{tabular}{|c|c|c|c|c|c|c|c|c|}
\hline \multirow[b]{2}{*}{ Variables } & \multicolumn{4}{|c|}{ Univariable Analysis } & \multicolumn{4}{|c|}{ Multivariable Analysis } \\
\hline & b (SE) & $\begin{array}{l}\text { Crude Hazards } \\
\text { Ratio }(95 \% \text { CI) }\end{array}$ & $\begin{array}{c}\text { Wald } \\
\text { Statistic }\end{array}$ & $\begin{array}{c}p- \\
\text { Value }\end{array}$ & b (SE) & $\begin{array}{c}\text { Adjusted } \\
\text { Hazards Ratio } \\
\text { (95\% CI) }\end{array}$ & $\begin{array}{c}\text { Wald } \\
\text { Statistic }\end{array}$ & $\begin{array}{c}p- \\
\text { Value }\end{array}$ \\
\hline $\begin{array}{l}\text { SOFA score on } \\
\text { AMS team } \\
\text { intervention day }\end{array}$ & & & & & & & & \\
\hline$\leq 4$ & 0 & 1 & & & 0 & 1 & & \\
\hline$>4$ & $1.63(0.26)$ & $5.11(3.06-8.54)$ & 6.22 & $<0.001$ & $1.88(0.27)$ & $6.61(3.90-11.18)$ & 7.03 & $<0.001$ \\
\hline CCS & & & & & & & & \\
\hline $0-2$ & & 1 & & & 0 & 1 & & \\
\hline$\geq 3$ & $0.84(0.26)$ & $2.32(1.40-3.86)$ & 3.26 & 0.001 & $0.67(0.26)$ & $1.97(1.17-3.30)$ & 2.57 & 0.01 \\
\hline
\end{tabular}

- Forward, backward, and stepwise Cox proportional hazards regression model applied.

- Multicollinearity and interactions were not observed. 
- The preliminary final model was properly specified.

- Hazard function plots, Log-minus-log plots, Schoenfeld partial residual plots, scaled and non-scaled Schoenfeld residuals test, and C-statistics were applied to check the assumption of the model.

- Regression diagnostics were performed by Cox-Snell residual, Martingale residual, deviance residual, and influential analysis.

- Influential outliers were identified by checking percent changes in regression coefficient.

\subsection{Impact of De-Escalation on 30-Day All-Cause Mortality}

Forced entry of AMS de-escalation into the final model (Table 9) indicated that patients de-escalated on extended or restricted antibiotics, whether early or late de-escalation, did not have a detrimental impact on 30-day all-cause mortality compared to continuation with extended and restricted antibiotics, after adjusting for confounders. The AHR for early and late de-escalation was 0.67 (95\% CI 0.36,1.22, $p=0.194)$ and $0.70(95 \%$ CI 0.35,1.41; $p=0.321$ ) respectively.

Table 9. Impact of antibiotic de-escalation after adjusting for SOFA score on intervention day and Charlson's comorbidity score.

\begin{tabular}{|c|c|c|c|c|}
\hline Variables & b (SE) & $\begin{array}{c}\text { Adjusted Hazards } \\
\text { Ratio }(95 \% \text { CI) }\end{array}$ & Wald Statistic & $p$-Value \\
\hline \multicolumn{5}{|c|}{$\begin{array}{l}\text { SOFA score on AMS team } \\
\text { intervention day }\end{array}$} \\
\hline$\leq 4$ & 0 & 1 & & \\
\hline$>4$ & $1.93(0.27)$ & $6.88(4.04-11.79)$ & 7.11 & $<0.001$ \\
\hline \multicolumn{5}{|l|}{ CCS } \\
\hline $0-2$ & 0 & 1 & & \\
\hline$\geq 3$ & $0.68(0.27)$ & $1.97(1.16-3.33)$ & 2.52 & 0.006 \\
\hline \multicolumn{5}{|l|}{ Types of intervention } \\
\hline No de-escalation & 0 & 1 & & \\
\hline Early de-escalation & $-0.40(0.31)$ & $0.67(0.36-1.22)$ & -1.30 & 0.194 \\
\hline Late de-escalation & $-0.35(0.35)$ & $0.70(0.35-1.41)$ & -0.99 & 0.321 \\
\hline
\end{tabular}

b: Regression coefficient; SE: Standard error; $\mathrm{HR}$ = hazard ratio; $\mathrm{CI}$ = confidence interval; SOFA = Sequential Organ Failure Assessment. Forced entry for primary variable of interest (Types of intervention) to adjust for SOFA score on intervention day and Charlson's comorbidity score. Multicollinearity and interactions were not observed.

\section{Discussion}

The overall rate of de-escalation in this study was $73 \%$. Several recent studies on de-escalation, most of which included mostly patients with identified pathogens, have documented de-escalation rates of 23-68\% [13-19]. In contrast to the aforementioned studies, the current study included patients with and without a microbiological diagnosis. Therefore, the de-escalation rate achieved $(73 \%)$ was slightly higher than those described in previous studies [13,20-22]. The higher overall rate of de-escalation, despite the absence of a microbiological diagnosis, may be explained by the presence of an AMS team in the hospital, who were available to prompt and recommend de-escalation to the primary team. The rate of de-escalation was also higher than that of intensive care unit (ICU) settings, as the current study involved less critically ill patients [8]. Early de-escalation in this study was slightly lower than that in the study by performed by Liu et al. [23], despite a similar hospital setting and the presence of an AMS team, due to varying definitions of de-escalation. When compared to studies with the same definition of de-escalation, the rates of early de-escalation and late de-escalation were similar to those in a study by Palacios-Baena, et al. [24]. The frequency of normal WBC counts $\left(<\times 10^{9} / \mathrm{L}\right)$ on day 0 and intervention was significantly higher in non-deescalated group than in early- and latedeescalated groups. This was because the primary team generally refuse to de-escalate once 
a patient has been shown to respond to an antibiotic regimen, as shown in normalization of white cell count, and would tend to continue and complete the antibiotic regimen.

Fleming-Harrington tests comparing overall mortality rates showed no significant difference between patients not de-escalated on extended or restricted antibiotics and those de-escalated early or later $(p=0.760)$. This result was in concordance with a study involving similar hospital settings conducted by Koupetori, et al. [25] on the survival of patients with bloodstream infection, in which log-rank test results were reported to be $p=0.683$. Two other studies conducted in ICU settings also generated similar results $[8,26]$.

Two variables were found to be associated with all-cause 30-day mortality of patients initiated with extended or restricted antibiotics: Charlson's comorbidity score (CCS) and SOFA score on AMS intervention day. The strength of the association for CCS was documented by Palacious-Baena et al. [24] with slight differences in AHR attributed to two reasons: first, the difference in CCS cut-offs, which is lower in our current study, and second, the difference in population diagnosis, for which Palacious-Baena et al. included only confirmed blood infection patients, while the current study included heterogeneous infection cases. SOFA scores $>4$ on the day of AMS intervention were also highly associated with mortality, reinforcing findings previously described in the literature, in which patient severity was an important factor in establishing prognosis after infection [27-30]. The strength of association was much higher compared to that in a study including only gramnegative bloodstream infection, in which it was found that patients with SOFA scores $>4$ have only double the risk of 30-day mortality with HR 2.18 (95\% CI 1.03, 4.57; $p=0.03)$ [21] Such a discrepancy may occur because SOFA scores were recorded at different time.

The overall mortality rates were not significantly different when patients were not de-escalated after controlling for confounders. The results from the current study are in accordance with findings from a prospective, multicenter cohort conducted by PalaciousBaena et al. The close similarity to the current study can most likely be attributed to several factors. First, the cut-off points for early and late de-escalation were similar for both studies. They also involved similar groups of antibiotics from which patients were de-escalated, namely imipenem, ertapenem, and meropenem. Third, the other study controlled for CCS and SOFA scores, which were also found to be associated with mortality in the current study. Similar results were again reiterated by Koupetori et al. [25] after controlling for confounders of septic shock/sepsis, age, gender, and concomitant disease.

To the best of our knowledge, this is the first Malaysian study that has focused on the impact of AMS de-escalation on patients using extended and restricted antibiotics. The interventions recommended by the AMS team did not compromise patient clinical outcome, which in this study is all-cause 30-day mortality.

This study has several strengths. First, it was conducted at the largest tertiary hospital under the Ministry of Health of Malaysia; hence, the results can be applied to other Malaysian hospitals with an AMS team. The mortality data were also derived from reliable documentation: the death registry and death certificate issued by Hospital Kuala Lumpur. Since the study had an objective clinical outcome that could be tracked, bias was not possible. One disadvantage of mortality as a clinical outcome is that if there are any changes in mortality, it is difficult to attribute those changes directly to an intervention. Hence, this study attempted to adjust for several confounders that could affect mortality, such as underlying comorbidities and severity of infection.

There are several limitations inherent to the design of this study. The retrospective design of our study is a methodological limitation, which is difficult to overcome because of the obvious ethical issues that must be considered when studying the management of a life-threatening illness. Analyzing patients in our cohort retrospectively may have resulted in the possibility of information bias and limited ability to study barriers to the de-escalation of extended and restricted antibiotics. Secondly, it is difficult to distinguish true pathogens from colonization. The suspicion of infection and the decision to obtain cultures, septic workup, or the choice and doses of antimicrobials depended mostly on the primary care physicians rather than being guided by a protocol or recommendations 
made by infectious disease specialists. In addition, this study assessed comorbidities retrospectively, which can result in an underestimation of their true prevalence. This study also involved a heterogeneous mix of infections and organisms that were included and analyzed collectively. Infections by these bacteria may potentially carry different risk factors and prognoses. The association between different mechanisms of resistance (e.g., AmpC, Extended beta-lactamases, Carbapenemese resistance) and outcomes of infection remains unclear in this study. Hence, it remains uncertain whether a de-escalation strategy can be implemented for infections caused by other, potentially antibiotic-resistant pathogens. Despite these shortcomings, we believe that the findings in this study establish several clinical variables that can help clinicians to identify patients at high risk of mortality.

This study has shown there is no difference in overall mortality if a patient is deescalated on extended or restricted antibiotics in medical wards. An association was found between the CCS and SOFA scores. These interesting observations could lead to further studies being conducted to understand the basis for these differences. Although CCS and SOFA scores are unmodifiable factors, understanding these differences and risk factors is important in the development of prediction models and personalized treatment. Practitioners can utilize such scores as a guide in the escalation of supportive therapy and other interventions, such as infection source control. If necessary, the family members of such groups can be informed regarding the chances of death during end-of-life or palliative care counseling.

For future studies, a bigger sample size is necessary so stratification according to the cause of death, either infection related or non-infection related, can be performed in addition to all-cause mortality. Since the current study is retrospective, the classification of mortality being either infection or non-infection related is difficult and can be biased; hence, a prospective study may overcome such a limitation. Despite limited evidence supporting the validity of mortality as a measure of stewardship programs, it remains an important patientcentered outcome. Future studies may consider investigating other clinically relevant outcomes, such as hospital readmission rates due to infection or recurrent infections, while disease-related mortality should primarily be used as a secondary or exploratory outcome. All-cause mortality can be reported in addition to disease-related mortality.

\section{Conclusions}

This study reinforces the fact that restricted or extended antibiotic de-escalation in patients does not have a detrimental impact on all-cause 30-day mortality compared to patients continued with restricted or extended antibiotics.

Author Contributions: H.L.T. designed data collection tools, monitored data collection for the whole trial, wrote the statistical analysis plan, cleaned and analysed data, and drafted and revised the paper; S.A. supervised administration of study and revised the paper; A.K.G. wrote the statistical analysis plan and revised the draft paper; R.A.K. and A.R. performed data collection, monitored data collection for the whole study and provided professional opinion. C.L.L. monitored data collection and provided professional opinion. All authors have read and agreed to the published version of the manuscript.

Funding: This research received no external funding.

Institutional Review Board Statement: The study was approved by the Human Research Ethics Committee Universiti Sains Malaysia (HREC) (Ref.: USM/JEPeM/19070392) with valid duration from 24 December 2019 until 23 December 2020 and the Medical Research and Ethics Committee of the Ministry of Health Malaysia (NMRR-19-2224-48672). The patient's identity was kept confidential by using random number identifiers that only known to the researcher. The identity of the patient will not be disclosed to third party other than an authorized individual. In this instance, the subjects' identity may be revealed to the USM, IRB/IEC, and the regulatory authority(ies) if applicable without violating the confidentiality of the subject, to the extent in accordance with the Guideline for Good Clinical Practice and Malaysian laws and regulations. Since this study or data collection was based entirely on data abstraction from existing medical or laboratory record; with no interaction with the 
human subject concerned and with no collection of identifiable private information, an informed consent is not required from the patient.

Informed Consent Statement: Not applicable.

Data Availability Statement: Data available on request due to restrictions eg privacy or ethical. The data that support the findings of this study are available on request from the corresponding author, The H.L. The data are not publicly available as data disclosure requires permission and ethical approval from Medical Research Ethical Committee (MREC), Malaysia.

Acknowledgments: The authors would like to thank the Director General of Health Malaysia for his/her permission to use data from health ministry hospital and also on the permission to publish this paper. We would also like to thank the antimicrobial stewardship programme members of Hospital Kuala Lumpur for continued assistance on this research.

Conflicts of Interest: The authors declare no conflict of interest.

\section{References}

1. Read, A.F.; Woods, R.J. Antibiotic resistance management. Evol. Med. Public Health 2014, 2014, 147. [CrossRef] [PubMed]

2. Ventola, C.L. The antibiotic resistance crisis: Part 1: Causes and threats. Pharm. Ther. 2015, 40, 277-283.

3. Friedman, N.; Temkin, E.; Carmeli, Y. The negative impact of antibiotic resistance. Clin. Microbiol. Infect. 2016, 22 , 416-422. [CrossRef]

4. Ministry of Health. Protocol on Antimicrobial Stewardship Program in Healthcare Facilities; Ministry of Health: Putrajaya, Malaysia, 2012.

5. Dellit, T.H.; Owens, R.C.; McGowan, J.E.; Gerding, D.N.; Weinstein, R.A.; Burke, J.P.; Huskins, W.C.; Paterson, D.L.; Fishman, N.O.; Carpenter, C.F.; et al. Infectious Diseases Society of America and the Society for Healthcare Epidemiology of America Guidelines for Developing an Institutional Program to Enhance Antimicrobial Stewardship. Clin. Infect. Dis. 2007, 44, 159-177. [CrossRef]

6. Kollef, M.H.; Kollef, K.E. Antibiotic Utilization and Outcomes for Patients with Clinically Suspected Ventilator-Associated Pneumonia and Negative Quantitative BAL Culture Results. Chest 2005, 128, 2706-2713. [CrossRef] [PubMed]

7. Rello, J.; Diaz, E. Pneumonia in the intensive care unit. Crit. Care Med. 2003, 31, 2544-2551. [CrossRef]

8. Khan, R.A.; Aziz, Z. A retrospective study of antibiotic de-escalation in patients with ventilator-associated pneumonia in Malaysia. Int. J. Clin. Pharm. 2017, 39, 906-912. [CrossRef] [PubMed]

9. Weiss, E.; Zahar, J.-R.; Lesprit, P.; Ruppe, E.; Leone, M.; Chastre, J.; Lucet, J.-C.; Paugam-Burtz, C.; Brun-Buisson, C.; Timsit, J.-F.; et al. Elaboration of a consensual definition of de-escalation allowing a ranking of $\beta$-lactams. Clin. Microbiol. Infect. 2015, 21, 649.e1-649.e10. [CrossRef]

10. Horan, T.C.; Andrus, M.; Dudeck, M. CDC/NHSN surveillance definition of health care-associated infection and criteria for specific types of infections in the acute care setting. Am. J. Infect. Control. 2008, 36, 309-332. [CrossRef] [PubMed]

11. Vincent, J.-L.; De Mendonça, A.; Cantraine, F.; Moreno, R.; Takala, J.; Suter, P.M.; Sprung, C.L.; Colardyn, F.; Blecher, S. Use of the SOFA score to assess the incidence of organ dysfunction/failure in intensive care units: Results of a multicenter, prospective study. Crit. Care Med. 1998, 26, 1793-1800. [CrossRef] [PubMed]

12. Vincent, J.L.; Moreno, R.; Takala, J.; Willatts, S.; De Mendonca, A.; Bruining, H.; Reinhart, C.K.; Suter, P.M.; Thijs, L.G. The SOFA (Sepsis-related Organ Failure Assessment) score to describe organ dysfunction/failure. Intensive Care Med. 1996, 22, 707-710. [CrossRef] [PubMed]

13. Alvarez-Lerma, F.; Alvarez, B.; Luque, P.; Ruiz, F.; Dominguez-Roldan, J.-M.; Quintana, E.; Sanz-Rodriguez, C. Empiric broadspectrum antibiotic therapy of nosocomial pneumonia in the intensive care unit: A prospective observational study. Crit. Care 2006, 10, R78. [CrossRef] [PubMed]

14. Carugati, M.; Franzetti, F.; Wiemken, T.; Kelly, R.; Peyrani, P.; Blasi, F.; Ramirez, J.; Aliberti, S. De-escalation therapy among bacteraemic patients with community-acquired pneumonia. Clin. Microbiol. Infect. 2015, 21, 936.e11-936.e18. [CrossRef] [PubMed]

15. Eachempati, S.R.; Hydo, L.; Barie, P.S. Gender-Based Differences in Outcome in Patients with Sepsis. Arch. Surg. 1999, 134, 1342-1347. [CrossRef] [PubMed]

16. Garnacho-Montero, J.; Gutiérrez-Pizarraya, A.; Escoresca-Ortega, A.; Corcia-Palomo, Y.; Fernández-Delgado, E.; Herrera-Melero, I.; Ortiz-Leyba, C.; Márquez-Vácaro, J.A. De-escalation of empirical therapy is associated with lower mortality in patients with severe sepsis and septic shock. Intensiv. Care Med. 2014, 40, 32-40. [CrossRef]

17. Gonzalez, L.; Cravoisy, A.; Barraud, D.; Conrad, M.; Nace, L.; Lemarié, J.; Bollaert, P.-E.; Gibot, S. Factors influencing the implementation of antibiotic de-escalation and impact of this strategy in critically ill patients. Crit. Care 2013, 17, R140. [CrossRef]

18. Viasus, D.; Simonetti, A.F.; Garcia-Vidal, C.; Niubó, J.; Dorca, J.; Carratalà, J. Impact of antibiotic de-escalation on clinical outcomes in community-acquired pneumococcal pneumonia. J. Antimicrob. Chemother. 2017, 72, 547-5553. [CrossRef]

19. Yamana, H.; Matsui, H.; Tagami, T.; Hirashima, J.; Fushimi, K.; Yasunaga, H. De-escalation versus continuation of empirical antimicrobial therapy in community-acquired pneumonia. J. Infect. 2016, 73, 314-325. [CrossRef] 
20. Apisarnthanarak, A.; Bhooanusas, N.; Yaprasert, A.; Mundy, L.M. Carbapenem De-escalation Therapy in a Resource-Limited Setting. Infect. Control. Hosp. Epidemiol. 2013, 34, 1310-1313. [CrossRef]

21. De Waele, J.J.; Ravyts, M.; Depuydt, P.; Blot, S.I.; Decruyenaere, J.; Vogelaers, D. De-escalation after empirical meropenem treatment in the intensive care unit: Fiction or reality? J. Crit. Care 2010, 25, 641-646. [CrossRef]

22. Lew, K.Y.; Ng, T.M.; Tan, M.; Tan, S.H.; Lew, E.L.; Ling, L.M.; Ang, B.; Lye, D.; Teng, C.B. Safety and clinical outcomes of carbapenem de-escalation as part of an antimicrobial stewardship programme in an ESBL-endemic setting. J. Antimicrob. Chemother. 2014, 70, 1219-1225. [CrossRef]

23. Liu, P.; Ohl, C.; Johnson, J.; Williamson, J.; Beardsley, J.; Luther, V. Frequency of empiric antibiotic de-escalation in an acute care hospital with an established Antimicrobial Stewardship Program. BMC Infect. Dis. 2016, 16, 751. [CrossRef]

24. Palacios-Baena, Z.R.; Delgado-Valverde, M.; Valiente Mendez, A.; Almirante, B.; Gomez-Zorrilla, S.; Borrell, N.; Corzo, J.E.; Gurgui, M.; de la Calle, C.; Garcia-Alvarez, L.; et al. Impact of de-escalation on prognosis of patients with bacteraemia due to Enterobacteriaceae: A post-hoc analysis from a multicenter prospective cohort. Clin. Infect. Dis. 2019, 69, 956-962. [CrossRef]

25. Koupetori, M.; Retsas, T.; Antonakos, N.; Vlachogiannis, G.; Perdios, I.; Nathanail, C.; Makaritsis, K.; Papadopoulos, A.; Sinapidis, D.; Giamarellos-Bourboulis, E.J.; et al. Bloodstream infections and sepsis in Greece: Over-time change of epidemiology and impact of de-escalation on final outcome. BMC Infect. Dis. 2014, 14, 272. [CrossRef] [PubMed]

26. Leone, M.; Bechis, C.; Baumstarck, K.; Lefrant, J.-Y.; Albanèse, J.; Jaber, S.; Lepape, A.; Constantin, J.-M.; Papazian, L.; Bruder, N.; et al. De-escalation versus continuation of empirical antimicrobial treatment in severe sepsis: A multicenter non-blinded randomized noninferiority trial. Intensiv. Care Med. 2014, 40, 1399-1408. [CrossRef] [PubMed]

27. Da Silveira, F.; Nedel, W.L.; Cassol, R.; Pereira, P.R.; Deutschendorf, C.; Lisboa, T. Acinetobacter etiology respiratory tract infections associated with mechanical ventilation: What impacts on the prognosis? A retrospective cohort study. J. Crit. Care 2019, 49, 124-128. [CrossRef] [PubMed]

28. Devran, O.; Karakurt, Z.; Adıgüzel, N.; Güngör, G.; Moçin, O.Y.; Balcı, M.K.; Celik, E.; Saltürk, C.; Takır, H.B.; Kargın, F.; et al. $\mathrm{C}$-reactive protein as a predictor of mortality in patients affected with severe sepsis in intensive care unit. Multidiscip. Respir. Med. 2012, 7, 47. [CrossRef]

29. Feng, D.-Y.; Zhou, Y.-Q.; Zhou, M.; Zou, X.-L.; Wang, Y.-H.; Zhang, T.-T. Risk Factors for Mortality Due to Ventilator-Associated Pneumonia in a Chinese Hospital: A Retrospective Study. Med Sci. Monit. Int. Med. J. Exp. Clin. Res. 2019, 25, 7660-7665. [CrossRef] [PubMed]

30. Inchai, J.; Pothirat, C.; Bumroongkit, C.; Limsukon, A.; Khositsakulchai, W.; Liwsrisakun, C. Prognostic factors associated with mortality of drug-resistant Acinetobacter baumannii ventilator-associated pneumonia. J. Intensiv. Care 2015, 3, 9. [CrossRef] [PubMed] 